\title{
Allografts in aortic position: Insights from a 27-year, single-center prospective study
}

\author{
Bardia Arabkhani, MD, MSc, Jos A. Bekkers, MD, PhD, Eleni-Rosalina Andrinopoulou, PhD, \\ Jolien W. Roos-Hesselink, MD, PhD, Johanna J. M. Takkenberg, MD, PhD, and \\ Ad J. J. C. Bogers, MD, PhD
}

\begin{abstract}
Objective: Over the past decades, the indication for allograft implantation in aortic position has evolved. The purpose of this study is to report long-term survival, allograft durability, and potential risk factors.

Methods: Between 1987 and 2010, 353 patients underwent aortic valve replacements via allograft (92 subcoronary, 261 root replacement; $98 \%$ aortic allografts). Patient characteristics, survival, valve durability, and valve-related events were analyzed. Patients also were followed with standardized echocardiography. A joint modeling approach was used to detect the effect of (echocardiographic) variables on mortality and reoperation hazard.
\end{abstract}

Results: Mean age was 45 years (range, 1 month to 84 years); $71 \%$ were males. The etiology was endocarditis in $32 \%$ (active $22 \%$ ), congenital $31 \%$, degenerative $9 \%$, aneurysm/dissection $12 \%$, rheumatic $6 \%$, and prosthetic valve failure $10 \%$. Hospital mortality was $5.9 \%(\mathrm{n}=21)$. During follow-up (mean 12 years, range, $0-24 ; 99 \%$ complete), 113 patients died. Twenty-year cumulative survival was $41 \%$ (95\% confidence interval, 32-50). Valve-related reoperations occurred in 117 patients: 100 structural valve deterioration, 9 nonstructural valve deterioration, and 8 endocarditis. Competing-risk analysis predicted that at 20 years $31 \%$ died, and $30 \%$ were alive without reoperation. Younger patient age was associated with increased reoperation. During follow-up left ventricular dilatation and severe aortic regurgitation were associated with mortality $(P=.006$ and .005 , respectively), and grade 3 or greater aortic regurgitation during follow-up was associated with risk of reoperation $(P=.001)$.

Conclusions: After almost 3 decades of experience with allografts in aortic position, the indication for use has become selective, mainly because of progressive structural valve deterioration over time. In case of complex aortic root pathology and active endocarditis allografts may still be useful. (J Thorac Cardiovasc Surg 2016;152:1572-9)

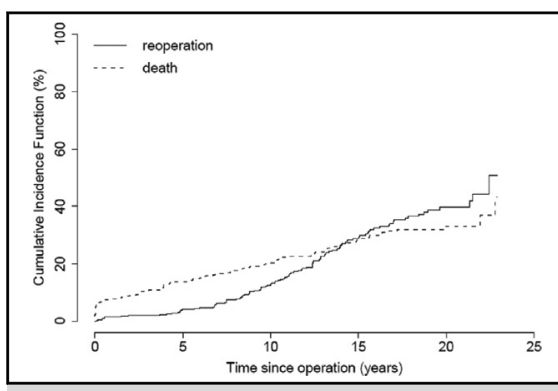

Freedom from reoperation for all patients, age subgroup, and surgical technique.

\section{Central Message}

Insights from a prospective study on allograft implantation in the aortic position, long term results.

\section{Perspective}

Allografts for aortic valve/root replacement yields excellent clinical results in the first postoperative decade, but in the second decade structural valve deterioration becomes apparent. In selected patients, especially patients with active endocarditis with complex aortic root pathology, allografts may still be considered, accepting an increased life-time risk of reoperation.

See Editorial Commentary page 1580.
The indication for implantation of allografts in aortic position has changed over the last decades. Initially allografts were regarded as a good alternative to biological and mechanical valve substitutes because of the excellent hemodynamics, low thrombo-embolic complications,

From the Erasmus University Medical Center, Rotterdam, The Netherlands.

Received for publication Nov 2, 2015; revisions received July 31, 2016; accepted for publication Aug 8, 2016.

Address for reprints: Bardia Arabkhani, MD, MSc, Department of Cardio-Thoracic Surgery, Bd 577, Erasmus Medical Centre, PO Box 2040, 3000 CA Rotterdam,

The Netherlands (E-mail: b.arabkhani@erasmusmc.nl).

$0022-5223 / \$ 36.00$

Copyright (c) 2016 by The American Association for Thoracic Surgery

http://dx.doi.org/10.1016/j.jtcvs.2016.08.013 good resistance to endocarditis, and no need for anticoagulation therapy. In addition, aortic allografts were found very useful in complex aortic root pathology with aortic annular destruction because of the flexible allograft tissue properties that allow for reconstruction of destructed tissue. The durability of the allografts, however, proved to be limited and age dependent, and the advantages of the allografts

Scanning this QR code will take you to the appendices for this article. 


\section{Abbreviations and Acronyms \\ $\mathrm{CI}=$ confidence interval \\ HR = hazard ratio \\ NSVD $=$ nonstructural valve deterioration \\ SVD = structural valve deterioration}

must be weighed against the risk of a possible reoperation attributable to structural valve deterioration (SVD) over time. $^{1,2}$

In 1987, we began a prospective cohort study in our institution using cryopreserved allografts for aortic valve and root disease. The allografts were used as a simple valve substitute, via a subcoronary implantation technique, as well as full-root replacement with reimplantation of the coronary arteries. Our earlier reports described the mid-term clinical outcome. ${ }^{3-5}$ After almost 3 decades, we are now able to present the long-term results of this prospective cohort. Comparable long-term follow-up has not been reported frequently.

The aim of our study is to describe the long-term clinical outcome after aortic valve and/or root replacement with allografts. In addition, the associations of several echocardiographic variables with survival and reintervention on implanted allograft are displayed.

\section{MATERIALS AND METHODS}

Between April 1987 and March 2013, 353 consecutive patients underwent allograft aortic valve or root replacement at Erasmus University Medical Center. All patients receiving an allograft in the aortic position are part of this prospective follow-up study in our center. ${ }^{3,6}$ Institutional review board approval was obtained for this study (MEC 12-477), and informed consent was waived.

\section{Operation Technique}

Cardiopulmonary bypass with moderate hypothermia was used in all surgical procedures. Crystalloid cardioplegia and topical cooling were used for myocardial protection. In addition, deep hypothermia and circulatory arrest were used in 37 patients with ascending aorta and/or arch pathology. Initially, the subcoronary technique was the preferred technique for aortic valve replacement, whereas from 1998 the root-replacement technique was performed (Appendix E1). A subcoronary technique initially was performed with scalloping of the sinuses of Valsalva $(n=32)$, whereas later on the noncoronary cusp was preserved $(n=63)$. Root replacement was performed as a freestanding root with reimplantation of the coronary arteries.

\section{Follow-up}

All patients who received an allograft (98\% aortic) at Erasmus University Medical Center were followed up prospectively through their visits to their cardiologist and by annual telephone interviews. In addition, echocardiographic follow-up was obtained at 6 months, 1 year postoperative, and thereafter biennially by means of serial standardized echocardiography. Valve-related events were registered according to the 2008 American Association for Thoracic Surgery/Society of Thoracic Surgeons/European Association for Cardiothoracic Surgery guidelines for reporting mortality and morbidity after cardiac valve interventions. ${ }^{7}$ The study database was frozen on December 31, 2014. Follow-up was $98 \%$ completed: 8 patients were lost to follow-up because of emigration.

\section{Statistical Methods}

Continuous data are presented as means (standard deviation; range). Comparison between groups was performed with an unpaired $t$ test unless data were not normally distributed (Mann-Whitney $U$ test in these cases). Categorical data are presented as proportions. Comparison was done by $\chi^{2}$ test or the Fisher exact test, where appropriate. The study started at the time of allograft implantation and ended at the time of event (death/reoperation) or at the last follow-up date. Univariable logistic regression analysis was used to study potential variables affecting early mortality (hospital and/ or 30-day mortality).The Cox proportional hazards model was used for univariable analyses of time-related events. Survival was analyzed with the Kaplan-Meier method (Appendix E2). Survival and freedom from overall reoperation were presented with the cumulative incidence function, from a competing risk analysis. Tests were performed 2 -sided, and a $P$ value of .05 was considered statistically significant. Variables that were tested as potential risk factors for hospital mortality, late mortality, and reoperation are displayed in Appendix E3.

For the analyses mentioned previously, Statistical Package for Social Sciences (SPSS) 21.0 for Windows statistical software (IBM Corp, Armonk, NY) was used. Advanced statistical linear mixed-effects model was used to assess changes in echocardiographic measurements of hemodynamic variables while accounting for the correlation between repeated measurements in each patient. Residuals plot was used to test the assumption of homoscedasticity in the model. Details of how the mixed-effects models were built are presented in Appendix E4. Furthermore, joint models of longitudinal and survival data were used to test whether echocardiographic variables were associated with survival or reoperation hazard. Specifically, this approach accounts for the biological variation in repeated (echocardiographic) measured variables within patients. ${ }^{8}$ The association of the following variables (which are of clinical interest) with survival and reoperation were tested: left ventricular end diastolic diameter (in $\mathrm{mm}$ ), left ventricular end systolic diameter (in $\mathrm{mm}$ ), sinotubular junction (in $\mathrm{mm}$ ), annulus diameter (in $\mathrm{mm}$ ), aortic regurgitation (grade), and aortic gradient (in $\mathrm{mm} \mathrm{Hg}$ ). For the advanced joint modeling $\mathrm{R}$ (version 3.1.3, available at: www.r-project.org) was used. A more detailed specification of the mixed-effects model and the joint model together with the syntax is provided in Appendix E4.

\section{RESULTS}

The mean follow-up was 11.5 years (range, $0-24.5$ years), with a total follow-up of 4188 patient years. There were several differences in characteristics between the subcoronary implantation and the full root replacement recipients. Table 1 displays patient characteristics and the preoperative data. Coronary artery bypass grafting as the result of complications related to reimplantation of the coronary arteries was necessary in 6 patients, of whom 2 subsequently died.

\section{Indication for Allograft Implantation}

The indication for implantation of an allograft has changed over the years. Between 1987 and 2005, 334 patients received an allograft in aortic position. The etiology of disease was $33 \%$ endocarditis; $23 \%$ prosthetic valve/allograft failure; $24 \%$ bicuspid valve disease; $7 \%$ rheumatic disease; $8 \%$ senile valve dysfunction; and $6 \%$ congenital disease. Between 2006 and 2014, the etiology 
TABLE 1. Patient characteristics and perioperative data

All patients $(\mathrm{n}=353)$

45 (16; 0.1-84)

72

Mean age, y (SD; range)

Male, \%

Creatinine, $\mu \mathrm{mol} / \mathrm{L}$ (SD; range)

Previous cardiac surgery

Hypertension

Ischemic heart disease

Connective tissue disease

Diabetes mellitus

Previous CVA

Ventilation support

Urgent operation $(<24 \mathrm{~h})$

Diagnosis

AR

AS

Combined AR + AS

Other

Etiology

Endocarditis

Active

Congenital (including bicuspid $\dagger$ )

Degenerative

Aneurysm

Rheumatic

Dissection

Other (prosthetic valves)

Systolic LVF

Good

Impaired

Moderate/severe

Preoperative NYHA class

I

II

III

IV

Perioperative characteristics

Valve requiring operation

Bicuspid

Tricuspid

Quadricuspid

Prosthesis

Allograft

Concomitant procedures

Aortic cross clamp time, min (SD; range)

Perfusion time, min (SD; range)

Circulatory arrest $(\mathrm{n}=35)$

$\min (\mathrm{SD}$; range)

Procedure-related CABG

Bleeding requiring reoperation

Permanent pacemaker

Perioperative CVA

Early mortality $(<30 \mathrm{~d})$
$102(85 ; 22-930)$

$26.3 \%(\mathrm{n}=93)$

$14.0 \%(\mathrm{n}=49)$

$8.5 \%(\mathrm{n}=30)$

$5 \%(\mathrm{n}=18)$

$3.4 \%(\mathrm{n}=12)$

$5.1 \%(\mathrm{n}=18)$

$5.9 \%(\mathrm{n}=21)$

$11 \%(\mathrm{n}=39)$

$79.4 \%(\mathrm{n}=201)$

$18.4 \%(\mathrm{n}=65)$

$17.5 \%(\mathrm{n}=62)$

$6.2 \%(\mathrm{n}=22)$

$32.5 \%(\mathrm{n}=115)$

$\mathrm{n}=80$

$31.1 \%(\mathrm{n}=111)$

$8.8 \%(\mathrm{n}=31)$

$7.1 \%(\mathrm{n}=25)$

$6.2 \%(\mathrm{n}=22)$

$5.1 \%(\mathrm{n}=18)$

$7.9 \%(\mathrm{n}=28)$

$74.2 \%(\mathrm{n}=267)$

$18.3 \%(\mathrm{n}=66)$

$6.6 \%(n=24)$

$25.8 \%(\mathrm{n}=92)$

$26.9 \%(\mathrm{n}=96)$

$29.7 \%(\mathrm{n}=106)$

$17.7 \%(\mathrm{n}=63)$

$36 \%(\mathrm{n}=130)$
$48 \%(\mathrm{n}=174)$
$1 \%(\mathrm{n}=2)$
$12 \%(\mathrm{n}=44)$
$1 \%(\mathrm{n}=4)$
$51 \%(\mathrm{n}=184)$
$142(58 ; 0-357)$
$198(78 ; 79-589)$
$4(14 ; 0-163)$
$2 \%(\mathrm{n}=6)$
$13 \%(\mathrm{n}=47)$
$4 \%(\mathrm{n}=16)$
$3 \%(\mathrm{n}=11)$
$5.9 \%(\mathrm{n}=21)$

Subcoronary technique $(n=92)$

Root replacement $(n=261)$

$46(16 ; 14-84)$
75

$113(107 ; 48-930)$

$17.4 \%(\mathrm{n}=16)$

$14.1 \%(\mathrm{n}=13)$

$10.8 \%(\mathrm{n}=10)$

0

$4.3 \%(\mathrm{n}=4)$

$8.6 \%(\mathrm{n}=8)$

0

$2.1 \%(\mathrm{n}=2)$

$56.7 \%(\mathrm{n}=52)$

$27.2 \%(\mathrm{n}=25)$

$16.1 \%(n=15)$

0

$33.6 \%(\mathrm{n}=31)$
$\mathrm{n}=13$

$32.6 \%(\mathrm{n}=30)$

$11.9 \%(\mathrm{n}=11)$$$
0
$$

$15.2 \%(\mathrm{n}=14)$

$$
0
$$

$6.5 \%(n=6)$

$78.9 \%(\mathrm{n}=75)$

$16.8 \%(\mathrm{n}=16)$

$4.3 \%(n=4)$

$12.6 \%(\mathrm{n}=12)$

$27.4 \%(\mathrm{n}=26)$

$48.4 \%(n=46)$

$11.6 \%(\mathrm{n}=11)$

$44 \%(\mathrm{n}=42)$

$47 \%(\mathrm{n}=45)$

0

$4 \%(\mathrm{n}=4)$

$3 \%(\mathrm{n}=3)$

$32 \%(\mathrm{n}=30)$

$132(30 ; 79-248)$

$176(40 ; 116-316)$

0

$$
\begin{gathered}
0 \\
14 \%(\mathrm{n}=13) \\
4 \%(\mathrm{n}=4) \\
3 \%(\mathrm{n}=3) \\
4.3 \%(\mathrm{n}=4)
\end{gathered}
$$

44 (17; 0.1-77)

71

$99(76 ; 22-900)^{*}$

$29.5 \%(\mathrm{n}=77)$

$13.8 \%(\mathrm{n}=36)$

$7.6 \%(\mathrm{n}=20)$

$6.8 \%(\mathrm{n}=18)^{*}$

$3.0 \%(\mathrm{n}=8)$

$3.8 \%(\mathrm{n}=10)$

$8 \%(\mathrm{n}=21)^{*}$

$14.2 \%(\mathrm{n}=37)$ *

$57.1 \%(\mathrm{n}=149)$

$15.3 \%(\mathrm{n}=40)$

$18.0 \%(\mathrm{n}=47)$

$8.4 \%(\mathrm{n}=22)$

$32.2 \%(\mathrm{n}=84)$

$\mathrm{n}=67$

$30.0 \%(\mathrm{n}=81)$

$7.6 \%(\mathrm{n}=20)$

$9.5 \%(\mathrm{n}=25) *$

$3.1 \%(\mathrm{n}=8)$

$6.9 \%(\mathrm{n}=18)$

$8.4 \%(\mathrm{n}=22)$

$72.5 \%(\mathrm{n}=192)$

$18.9 \%(\mathrm{n}=50)$

$7.5 \%(\mathrm{n}=20)$

$30.5 \%(\mathrm{n}=80)$

$26.7 \%(\mathrm{n}=70)$

$22.9 \%(\mathrm{n}=60)$

$19.8 \%(\mathrm{n}=52)$

$33 \%(\mathrm{n}=88)$

$49 \%(\mathrm{n}=129)$

$1 \%(n=2)$

$15 \%(\mathrm{n}=40)^{*}$

$1 \%(\mathrm{n}=3)$

$58 \%(\mathrm{n}=154)^{*}$

145 (65; 0-357)

206 (86; 79-589)

5 (17; 0-163)

$2 \%(\mathrm{n}=6)$

$13 \%(\mathrm{n}=34)$

$5 \%(\mathrm{n}=12)$

$3 \%(\mathrm{n}=8)$

$6.5 \%(\mathrm{n}=17)$

$S D$, Standard deviation; $C V A$, cerebrovascular accident; $A R$, aortic valve regurgitation; $A S$, aortic valve stenosis; $L V F$, left ventricular function; NYHA, New York Heart Association; $C A B G$, coronary artery bypass grafting. *Statistical significant difference between the 2 groups (unpaired $t$ test or Mann-Whitney $U$ test) $\dagger$ Endocarditis excluded. 


\section{Etiology of disease}

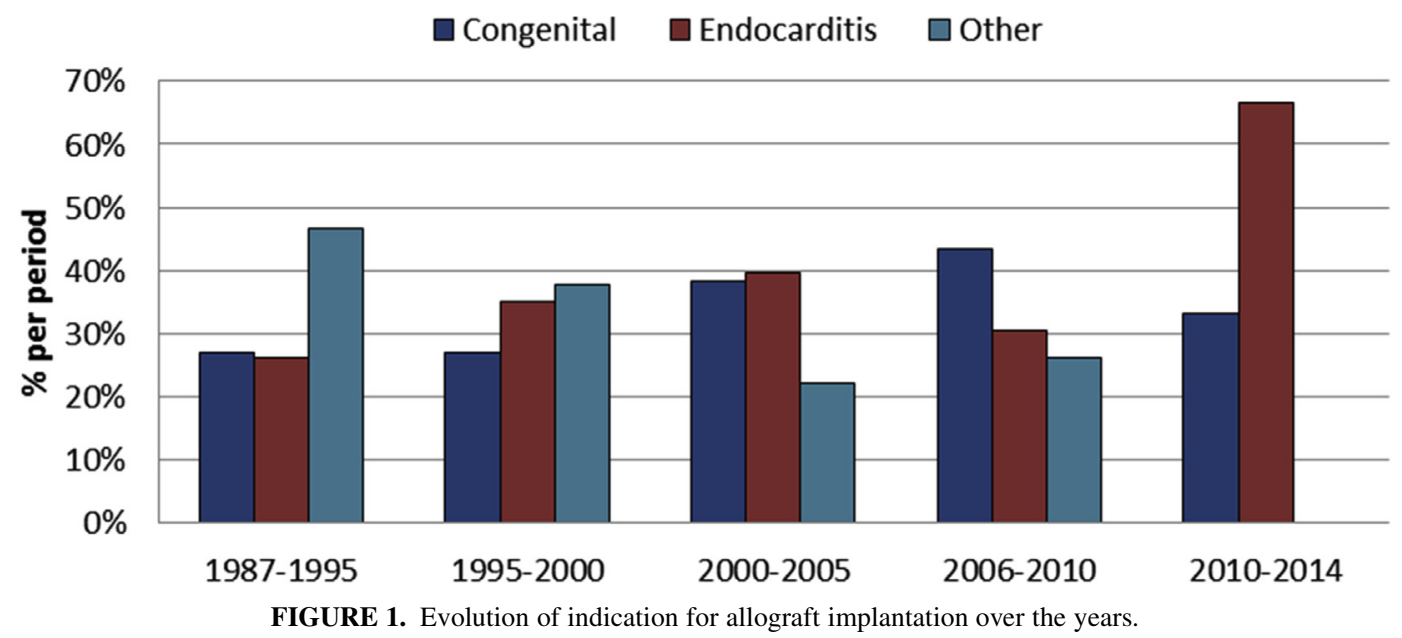

of disease was $(n=26): 41 \%$ bicuspid valve disease; $35 \%$ endocarditis; $12 \%$ senile valve dysfunction; $8 \%$ prosthetic valve/allograft failure; and 4\% rheumatic disease. Figure 1 displays the etiology of disease and thus the indication for allograft implantation over the years. Allograft characteristics are displayed in Appendix E5.

\section{Early Mortality and Morbidity}

Operative mortality concerned 6 patients and was attributed to persistent massive bleeding in 3 patients ( 1 had an active endocarditis with abscesses, 1 with an acute dissection, and 1 patient who underwent a reoperation for paravalvular leakage of a mechanical valve), left ventricular failure in 2 patients (1 patient with an active prostheticvalve endocarditis and 1 patient with acute endocarditis including a fistula to the left atrium), and finally 1 patient who died during a salvage procedure of a prosthetic aortic valve endocarditis with extensive tissue destruction of the left ventricular outflow tract and proximal ascending aorta with abscesses. Another 15 patients died within 30 days postoperatively or during the same hospitalization. The causes of death were registered as cardiac and not valverelated in 10 patients. Three valve-related early deaths concerned 2 patients who died of a major intracerebral bleeding and 1 patient with a myocardial infarction caused by kinking of the reimplanted right coronary artery. Another patient had an acute endocarditis and died as a result of a stroke caused by septic emboli, and finally 1 patient who was operated on because of an active endocarditis of a biological valve substitute who developed multiorgan failure and died. Early mortality was $5.8 \%$.

In 6 patients, additional coronary artery bypass grafting for complications related to reimplantation of the coronary arteries was necessary, of which 2 subsequently died. In 1 patient, coronary orifice stenosis occurred because the left coronary artery button was too small. Another patient had annular calcifications extending up to the right coronary ostium that was qualitatively poor and ruptured after reimplantation. A third patient had an active bioprosthetic endocarditis with abscesses, and after reimplantation the edematous right coronary artery button ruptured. Two other patients experienced right ventricular dysfunction due to kinking of the reimplanted right coronary artery. In another patient, the coronary artery buttons were large, possibly causing malperfusion of the right and left coronary artery.

\section{Late Survival}

During follow-up, another 113 patients died. There were 72 nonvalve-related deaths. In addition, 42 patients died from a valve-related cause: 26 patients died sudden, unexpected, and unexplained; 5 patients died from a major bleeding (3 of them were on anticoagulation therapy because of atrial fibrillation in 2 and a mechanical mitral valve prosthesis in 1 patient); 4 patients who had structural (allograft) valve deterioration died of heart failure; 3 patients died due to endocarditis; and another patient died after a cerebrovascular accident. The cause of death could not be retrieved in 3 patients. Cumulative survival (including early mortality) was $98.20 \%$ (95\% confidence interval [CI], 96\%-99\%) at 1 year, 87.5\% (95\% CI, 83\%-90\%) at 10 years, and $40.0 \%(95 \% \mathrm{CI}, 32 \%-50 \%)$ at 20 years respectively. Figure 2 shows the Kaplan-Meier curve of cumulative late survival, including early mortality. Patients subsequently were subdivided in 3 age categories: (1) 0 to 20 years at operation, (2) 21 to 60 years at operation, and (3) 61 years and older. Patients in the latter category have a significant worse survival compared with other categories. Additionally, multivariable (independent) predictors of late 


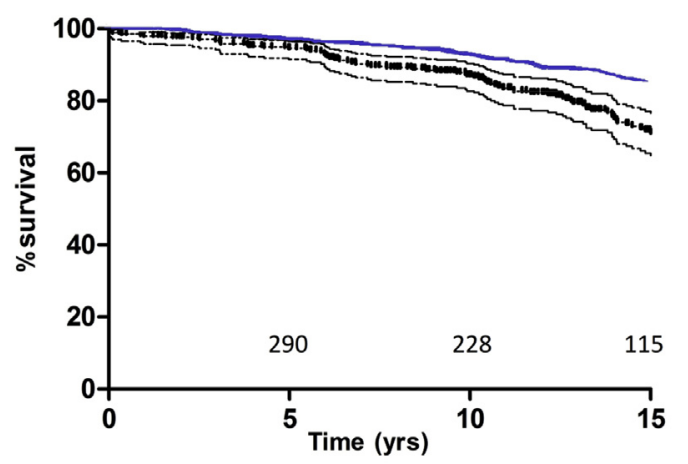

Age categories:

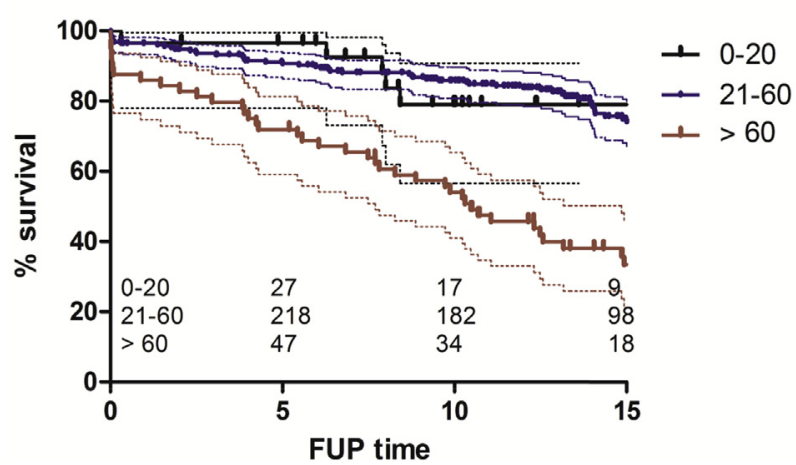

FIGURE 2. Top, Cumulative survival (all patients) including $95 \%$ CI. Blue line indicates general Dutch population (mean age 45 years). Bottom, Survival by subgroup. Upper line indicates patients aged 0 to 20 years; Middle line indicates patients aged from 21 to 60 years; lower line indicates patients aged 61 and older. Tyrone-Ware test shows a significant $(P \leq .001)$ worse survival in patients aged $>50$ years at OR. No. at risk are displayed in beneath the black box. FUP, Follow-up.

mortality in our Cox model were patient age at operation (hazard ratio [HR], 1.06, 95\% CI, 1.03-1.08; $P<.001$ ) and preoperative ventilation support (HR, $2.58,95 \% \mathrm{CI}$ $1.20-5.32 ; P=.01)$.

\section{Competing Risk for Death or Subsequent Reoperation}

A total of 110 patients were reoperated on because of an allograft related cause. Reason for reoperation was SVD in 93 patients, nonstructural valve deterioration (NSVD) in 9 patients, and endocarditis in 8 patients. The allograft was replaced by a mechanical valve substitute in 62 patients, a composite valve replacement (Bentall procedure) in 31 patients, allograft in 3 patients, autograft in 4 patients, stented bioprosthesis in 12 patients, and transcatheter aortic valve implantation in 2 patients. In 1 patient, the allograft could be saved by removal of vegetation from the proximal anastomosis of the allograft 3 weeks after the initial operation because of active endocarditis. Ten years later, this allograft was replaced with a mechanical valve. In another patient, a false aneurysm of the allograft was closed operatively. Finally, in another patient a fistula from the allograft towards the right atrium was closed. Reoperation mortality was $3.9 \%$. During follow-up 99 patients died without a reoperation and 104 were reoperated who were still alive at last follow-up date. Competing-risks analysis predicted that after 20 years from initial allograft implantation, $31 \%$ had died without a reoperation, $39 \%$ underwent a reoperation, and $30 \%$ remained alive without reoperation (Figure 3).

\section{Valve-Related Complications}

SVD and NSVD. A total of 98 patients experienced SVD. Replacement of the allograft due to SVD was performed in 100 patients. The other 4 patients died due to SVD while being treated medically. SVD was due mainly to the calcification of the aortic (allograft) root in the second decade of follow-up. The progression of aortic gradient in time is displayed in Figure 4. NSVD occurred in 9 patients, and it was mainly due to (paravalvular) leakage as a result of technical error in patients who received an allograft using the subcoronary implantation technique.

Endocarditis. There were 14 cases of endocarditis. Endocarditis was the reason for reoperation on the allograft in 8 patients. In addition, 3 other patients were diagnosed with endocarditis, 2 of whom were treated medically without the need of reoperation, and 1 patient was a drug user who could not be operated because of brain abscesses and was treated medically and died eventually. As described in the section "Late Survival," 3 other patients died from endocarditis. Reoccurrence of endocarditis occurred in 6 patients. In 4 of these patients there was an active endocarditis noticed at the operation time of allograft implantation.

\section{Other Valve-Related Complications}

During follow-up 20 cerebrovascular events were registered, 1 patient died because of a cerebrovascular accident, as described previously. Besides the 5 lethal bleedings described earlier, there was another (nonlethal) major bleeding.

Echocardiographic variables. A subset of 308 patients was followed by means of standardized echocardiography with a mean of 6 echocardiograms (range, 1-13). Variables in the joint model predicting greater hazard of mortality were: severe (grade 3 and/or 4) aortic regurgitation (HR, 1.06; CI, 1.0-1.1; $P=.03$ ) and enlargement of left ventricle end systolic dimension (HR, 1.05; CI, 1.01-1.09; $P=.007$ ). Variables predicting (joint model) a greater reoperation hazard were: progression of the gradient over the allograft (HR, 1.40; CI, 1.13-1.76; $P=.001$ ); progression of allograft valve regurgitation (HR, 1.05; $\mathrm{CI}, 1.01-1.09 ; P=.005)$, and increase in aortic annulus diameter (HR, 2.33; CI, $0.99-5.88 ; P=.053)$. 


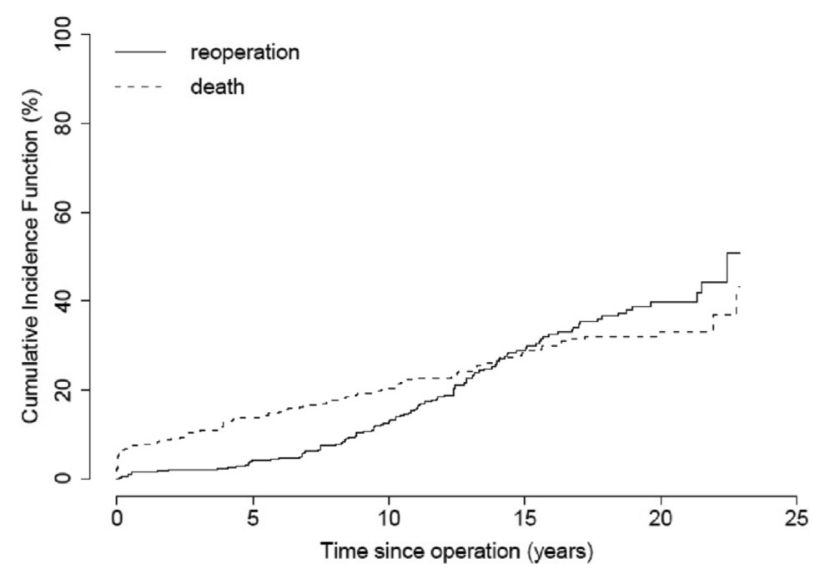

FIGURE 3. Competing-risks analysis for subsequent reoperation on the allograft or death. All patients were included at the time of initial allograft implantation $(n=353)$ and could transition to either death or a subsequent reoperation on the allograft.

There is a progression of aortic valve (ie, allograft) regurgitation (Appendix E6). Largely patients with grade 1 aortic regurgitation progress to grade 2 aortic regurgitation over time. Mixed effect modeling reveals the following predictors to be correlated with progression of regurgitation over the aortic allograft: time from operation $(P \leq .001)$, subcoronary technique $(P \leq .001)$, and active endocarditis $(P=.02)$.

\section{DISCUSSION}

After almost 3 decades, we observe good long-term outcome after allograft implantation with regard to mortality and occurrence of thromboembolic and hemorrhagic events; however, the risk of a reoperation due to SVD in the second decade after allograft implantation is increased. Furthermore, survival and freedom from reoperation is roughly comparable with other biological valve substitutes. ${ }^{1}$ Despite a decrease of implantation of allografts in aortic position over the years, use in selected cases of active aortic root endocarditis and complex aortic root pathology may still be useful.

In the 1980s enthusiasm existed for the use of allografts for aortic valve or root replacement as a superior durability of human tissue valves compared with bioprostheses was envisioned; however, with time it became evident that allograft durability was not better but more or less comparable with bioprostheses and inferior to mechanical prosthesis. ${ }^{1,9}$ This finding is reflected in the current European Society of Cardiology/European Association for Cardio-Thoracic Surgery guidelines for the management of patients with valvular heart disease that have no specific recommendations in favor of allografts for aortic valve replacement, except for active endocarditis with perivalvular lesions. ${ }^{10}$

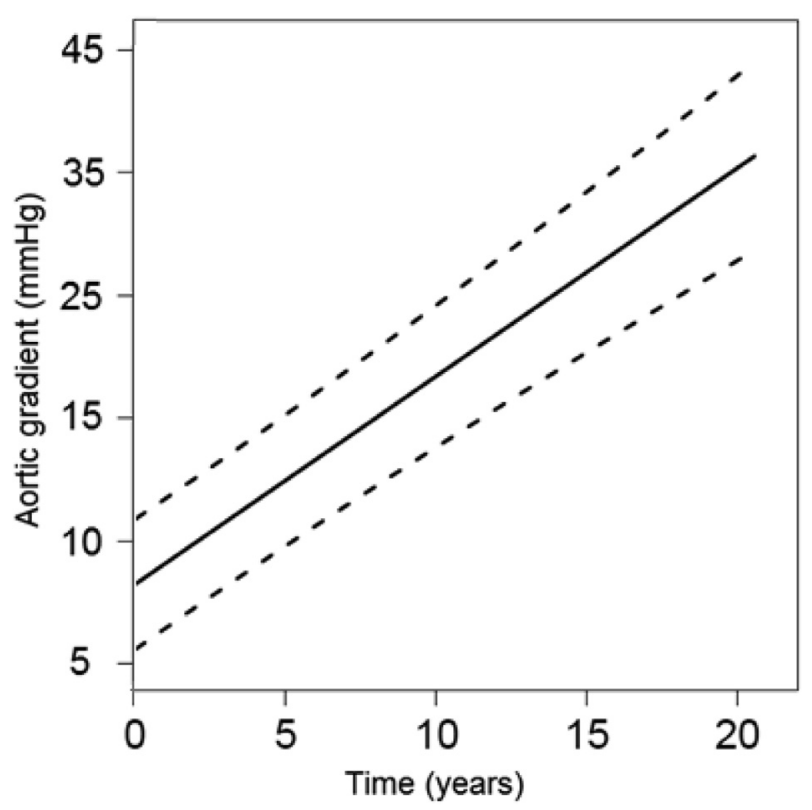

FIGURE 4. Progression of aortic gradient over time. The dashed lines denote $95 \%$ confidence intervals.

\section{Survival and Reoperation}

Our findings regarding patient survival and reoperation rates show good results even after 3 decades of follow-up, with median survival of around 20 years. Patient survival is comparable with patients after a biological valve in aortic position; however, there seems to be a slightly greater hazard of reoperation in allografts. This was also shown in a clinical trial by El-Hamamsy and colleagues ${ }^{9}$ in which they compared allografts with freestyle biological valve substitute. Of note, the patient characteristics in the study of El Hamamsy and colleagues differed from ours, as there were just a few patients with endocarditis as etiology of disease in their randomized control trial. Valve-related event occurrence such as SVD and reoperation hazard is comparable with previous reports. ${ }^{11,12}$

Additionally, another option for the treatment of aortic valve disease, especially in younger patients, is the Ross procedure. Some great results have been achieved, with freedom from reoperation of about $90 \%$ to $99 \%$ at 15 years of follow-up ${ }^{13,14}$; however, these results have only been achieved in slightly different patients groups and in highly specialized centers.

\section{SVD and Mode of Failure}

Early valve failure occurred mainly in the subcoronary implanted allografts in the first few years after implantation and was mainly due to technical errors during the implantation. The suboptimal results of the subcoronary implantation technique were noticed and therefore we started to use the subcoronary technique less frequently and stopped using it after 1998. 
If one disregards the early technical failures in the subcoronary allografts, then the pattern of SVD is comparable with root replacement allografts in the first postoperative decade. ${ }^{11}$ In the second postoperative decade, it appears that subcoronary implants have a slightly better SVD pattern compared with root replacement allografts, but the low number of patients still at risk at that point in time prohibits any firm conclusions.

In long-term follow-up, the main reason for reoperation was SVD in most cases. SVD is known to be the main cause of allograft failure. ${ }^{3,15}$ During reoperation, we noticed that in most cases the allograft sinuses of Valsalva were calcified, with or without fenestration/rupture of the valve leaflets, causing valvular stenosis and/or regurgitation. Although we know from the literature that a reoperation after allograft implantation can be challenging and associated with greater hazards of less favorable outcome, ${ }^{15,16}$ hospital mortality was relatively low in our patients, as described previously by Bekkers and colleagues. ${ }^{5}$ Notably, almost all reoperations were performed by the same surgeon, and this expert experience could be of influence in obtaining these excellent results. Our rigorous follow-up regimen with annual clinical and biennial echocardiographic standardized follow-up also allowed us to detect allograft failure early on and carefully plan for an elective reoperation in most cases.

\section{Other Valve-Related Complications}

The occurrence of endocarditis was low, as expected, and the main indication for allograft use nowadays. ${ }^{17,18}$ In addition, the reoccurrence of endocarditis in patients who had received an allograft because of infective endocarditis was low. This finding should be taken into consideration when looking for a suitable valve substitute to treat an infective endocarditis in aortic position, especially with complex aortic root pathology with abscesses and/or fistula formation around the root. In these cases it may be difficult to use the Bentall prosthesis to cover the entire aortic root without leaving behind cavities and is an allograft a good alternative.

Thromboembolic event occurrence was uncommon, highly favorable in comparison with mechanical valve prosthese $^{19}$ and comparable with biological valve prosthesis $^{4,9,20}$ and underlines once more the advantages of biological valve substitutes in this regard. Hemorrhagic events occurred in only 5 patients, of whom 3 were on anticoagulation therapy, 2 patients because of atrial fibrillation and 1 due to earlier implanted mitral valve prosthesis. The avoidance of anticoagulation therapy in allograft aortic valve or root replacement is particularly important for patients with an active lifestyle as well as in female patients who have a desire of future pregnancy, especially given the notion that pregnancy is not associated with allograft failure. ${ }^{21}$

\section{Echocardiographic Outcome}

We found that enlargement of left ventricle end systolic dimension and progression of aortic (allograft) regurgitation is associated with a greater mortality hazard probably due to less favorable hemodynamics. Additionally, an increase in aortic annulus diameter; progression of the gradient over the allograft, and progression of allograft regurgitation is associated with a greater reoperation hazard. This information should help to be able to intervene earlier in the process in order to adjust treatment where possible, and to help evaluate the patients at risk more accurately and maybe more frequently, certainly in the second decade after allograft implantation where SVD play a significant role. The challenging goal is still to find out which specific dynamic variables and variations are associated with worse outcome, and what exactly the timing for (surgical) intervention must be.

To estimate the association between a single measure and time to event, standard statistical tools such as Cox regression are applicable; however, when it comes to the analysis of repeated measurements in relation to time-to-event, the Cox model including time-dependent covariates, has been used widely, although problems arise from the fact that repeated measurements may contain biological variation that is not taken into account by the time-dependent (covariates) Cox model. The problem with ignoring this biologic variation and using the time-dependent covariates Cox model is that derived results may be substantially biased. Therefore the joint model for longitudinal and survival data have been proposed. Despite the appropriateness of these models, there are some disadvantages. The joint models of longitudinal and survival data are not easily applicable by any physician since a level of expertise in programming may be required. The complexity of analyses of SVD and risk factors related to an increased risk of SVD is addressed recently by Blackstone, ${ }^{22}$ and the importance and usefulness of such models have been demonstrated. ${ }^{23,24}$ More recently there are more statistical tools available as the joint modeling approach for the prognostic evaluation of serial biomarkers, ${ }^{25}$ specifically the concept of joint modeling of longitudinal and survival data in repeated valve function measurements after implantation of an allograft is introduced by Andrinopoulou and colleagues ${ }^{8}$ in 2012. The translation of the longitudinal data to a predictive-model using thesestatistical tools should allow us to understand the association between the serial measured parameters influencing SVD (ie, aortic gradient, aortic regurgitaion, verntricul dimentions, etc), and important clinical endpoints such as survival and reoperation.

\section{Limitations}

Our study concerns a single-center experience with a heterogeneous etiology of disease, with a relatively large 
proportion of patients with endocarditis, and furthermore significant differences between patients receiving allografts as a subcoronary implant compared with recipients with full root replacement. This may lead to interpretations that are not necessarily applicable to other patient cohorts.

\section{CONCLUSIONS}

Implantation of an allograft in aortic position is associated with low valve-related events perioperatively. From this prospective observational cohort study that spans almost 30 years, it becomes evident that although allograft aortic valve or root replacement yields excellent clinical results in the first postoperative decade, in the second postoperative decade an SVD pattern that resembles bioprostheses becomes apparent. Reoperation on degenerated allografts proofs challenging but can be managed well through systematic clinical and echocardiographic follow-up and careful planning of elective reoperation. In selected patients, especially those less favorable for anticoagulation therapy, and patients with active endocarditis with complex aortic root pathology, allografts may still be considered, accepting an increased life-time risk of reoperation.

\section{Conflict of Interest Statement}

Authors have nothing to disclose with regard to commercial support.

\section{References}

1. Nowicki ER, Pettersson GB, Smedira NG, Roselli EE, Blackstone EH, Lytle BW. Are allografts the biologic valve of choice for aortic valve replacement in nonelderly patients? Comparison of explantation for structural valve deterioration of allograft and pericardial prostheses. J Thorac Cardiovasc Surg. 2006;131:558-64.e4.

2. Takkenberg JJ, Eijkemans MJ, van Herwerden LA, Steyerberg EW, Lane MM, Elkins RC, et al. Prognosis after aortic root replacement with cryopreserved allografts in adults. Ann Thorac Surg. 2003;75:1482-9.

3. Takkenberg JJ, van Herwerden LA, Eijkemans MJ, Bekkers JA, Bogers AJ. Evolution of allograft aortic valve replacement over 13 years: results of 275 procedures. Eur J Cardiothorac Surg. 2002;21:683-91; discussion 691.

4. Takkenberg JJ, Klieverik LM, Bekkers JA, Kappetein AP, Roos JW, Eijkemans MJ, Bogers AJ. Allografts for aortic valve or root replacement: insights from an 18-year single-center prospective follow-up study. Eur $J$ Cardiothorac Surg. 2007;31:851-9.

5. Bekkers JA, Klieverik LM, Raap GB, Takkenberg JJ, Bogers AJ. Re-operations for aortic allograft root failure: experience from a 21-year single-center prospective follow-up study. Eur J Cardiothorac Surg. 2011;40:35-42.

6. Takkenberg JJ, vanHerwerden LA, Galema TW, Bekkers JA, KleyburgLinkers VE, Eijkemans MJ, Bogers AJ. Serial echocardiographic assessment of neo-aortic regurgitation and root dimensions after the modified Ross procedure. J Heart Valve Dis. 2006;15:100-6; discussion 106-7.

7. Akins CW, Miller DC, Turina MI, Kouchoukos NT, Blackstone EH, Grunkemeier GL, et al. Guidelines for reporting mortality and morbidity after cardiac valve interventions. Ann Thorac Surg. 2008;85:1490-5.

8. Andrinopoulou ER, Rizopoulos D, Jin R, Bogers AJ, Lesaffre E, Takkenberg JJ. An introduction to mixed models and joint modeling: analysis of valve function over time. Ann Thorac Surg. 2012;93:1765-72.
9. Smedira NG, Blackstone EH, Roselli EE, Laffey CC, Cosgrove DM. Are allografts the biologic valve of choice for aortic valve replacement in nonelderly patients? Comparison of explantation for structural valve deterioration of allograft and pericardial prostheses. J Thorac Cardiovasc Surg. 2006;131:558-64.

10. Sarang Z, Melina G, Takkenberg JJ, Yacoub MH. Late outcomes following freestyle versus homograft aortic root replacement: results from a prospective randomized trial. J Am Coll Cardiol. 2010;55:368-76.

11. Vahanian A, Alfieri O, Andreotti F, Antunes MJ, Baron-Esquivias G, Baumgartner $\mathrm{H}$, et al. Guidelines on the management of valvular heart disease (version 2012): the Joint Task Force on the Management of Valvular Heart Disease of the European Society of Cardiology (ESC) and the European Association for Cardio-Thoracic Surgery (EACTS). Eur J Cardiothorac Surg. 2012;42:S1-44.

12. Lund O, Chandrasekaran V, Grocott-Mason R, Elwidaa H, Mazhar R, Khaghani A, et al. Primary aortic valve replacement with allografts over twenty-five years: valve-related and procedure-related determinants of outcome. J Thorac Cardiovasc Surg. 1999;117:77-90; discussion 90-1.

13. O'Brien MF, Harrocks S, Stafford EG, Gardner MA, Pohlner PG, Tesar PJ, Stephens F. The homograft aortic valve: a 29-year, 99.3\% follow up of 1,022 valve replacements. J Heart Valve Dis. 2001;10:334-44; discussion 335.

14. Skillington PD, Mokhles MM, Wilson W, Grigg L, Larobina M, O’Keefe M, Takkenberg J, et al. Inclusion cylinder method for aortic valve replacement utilising the Ross operation in adults with predominant aortic stenosis - $99 \%$ freedom from re-operation on the aortic valve at 15 years. Glob Cardiol Sci Pract. 2013;2013:383-94.

15. da Costa FD, Takkenberg JJ, Fornazari D, Balbi Filho EM, Colatusso C, Mokhles MM, et al. Long-term results of the Ross operation: an 18-year single institutional experience. Eur J Cardiothorac Surg. 2014;46:415-22; discussion 422.

16. Joudinaud TM, Baron F, Raffoul R, Pagis B, Vergnat M, Parisot C, et al. Redo aortic root surgery for failure of an aortic homograft is a major technical challenge. Eur J Cardiothorac Surg. 2008;33:989-94.

17. Habib G, Hoen B, Tornos P, Thuny F, Prendergast B, Vilacosta I, et al. Guidelines on the prevention, diagnosis, and treatment of infective endocarditis (new version 2009): the Task Force on the Prevention, Diagnosis, and Treatment of Infective Endocarditis of the European Society of Cardiology (ESC). Endorsed by the European Society of Clinical Microbiology and Infectious Diseases (ESCMID and the International Society of Chemotherapy (ISC) for Infection and Cancer. Eur Heart J. 2009;30:2369-413.

18. Byrne JG, Rezai K, Sanchez JA, Bernstein RA, Okum E, Leacche M, et al Surgical management of endocarditis: the society of thoracic surgeons clinical practice guideline. Ann Thorac Surg. 2011;91:2012-9.

19. Cannegieter SC, Rosendaal FR, Briet E. Thromboembolic and bleeding complications in patients with mechanical heart valve prostheses. Circulation. 1994;89:635-41.

20. Rahimtoola SH. Choice of prosthetic heart valve for adult patients. J Am Coll Cardiol. 2003;41:893-904.

21. Arabkhani B, Heuvelman HJ, Bogers AJ, Mokhles MM, Roos-Hesselink JW, Takkenberg JJ. Does pregnancy influence the durability of human aortic valve substitutes? J Am Coll Cardiol. 2012;60:1991-2.

22. Blackstone EH. Of SOLOs and ensembles. J Thorac Cardiovasc Surg. 2015;150 $10-1$.

23. Mokhles MM, Rajeswaran J, Bekkers JA, Borsboom GJ, Roos-Hesselink JW, Steyerberg EW, et al. Capturing echocardiographic allograft valve function over time after allograft aortic valve or root replacement. J Thorac Cardiovasc Surg. 2014;148:1921-8.e3.

24. Blackstone EH. Breaking down barriers: helpful breakthrough statistical methods you need to understand better. J Thorac Cardiovasc Surg. 2001;122: 430-9.

25. Rizopoulos D. Joint Models for Longitudinal and Time-to-Event Data with Ap plications in R. Boca Raton: Chapman and Hall/CRC Biostatistics Series; 2012.

Key Words: allograft(s), homograft(s), aortic valve replacement, aortic root, biological valve 


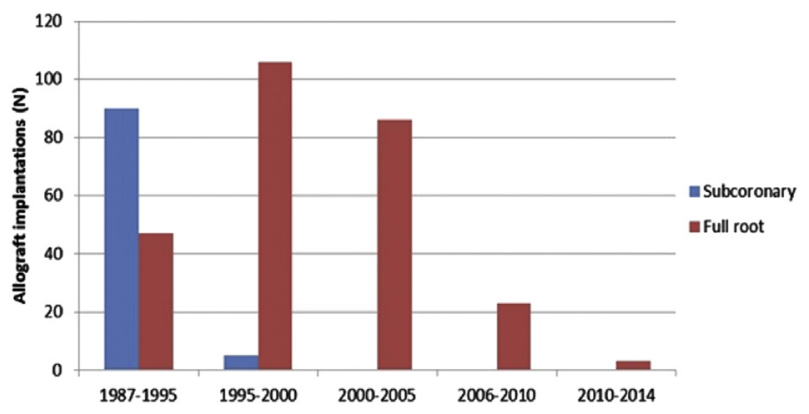

APPENDIX E1. Number of allografts and the surgical technique used for implantation by year of operation.

APPENDIX E3. Variables that were tested (univariable) as potential risk factors for hospital mortality, late mortality, and reoperation

\begin{tabular}{ll}
\hline \multicolumn{1}{c}{ Variable } & \multicolumn{1}{c}{ Specification } \\
\hline Patient age & Continuous variable $(\mathrm{y})$ \\
Sex & \\
Preoperative ventilation support & \\
$\begin{array}{l}\text { Preoperative abnormal cardiac } \\
\text { rhythm }\end{array}$ & Any rhythm other that sinus rhythm \\
Preoperative renal function & Creatinine, continuous (mmol/L)
\end{tabular}

Severe renal disease requiring either dialysis or transplantation

Prior cardiac surgery

Marfan disease

Ischemic heart disease

Etiology of heart valve disease

Preoperative hypertension

Systolic left ventricular function

Previous cerebrovascular accident

Preoperative New York Heart

Good/impaired/moderate/bad

Association class

From I to IV

Emergency of the procedure

Cardiopulmonary bypass time Continuous ( $\mathrm{min})$

Hemodynamic diagnosis*

Allograft diameter

Continuous $(\mathrm{mm})$

*Exclusively for reoperation.

APPENDIX E2. Potential univariable risk factors for increased early and late mortality

\begin{tabular}{lrcr}
\hline \multicolumn{1}{c}{ Variable } & HR & $\mathbf{9 5} \%$ CI & $\boldsymbol{P}$ value \\
\hline Early mortality & & & \\
Age & 1.1 & $1.0-1.1$ & $<.001$ \\
Active Endocarditis & 3.8 & $1.6-9.2$ & .003 \\
Severe renal disease & 13.7 & $3.9-47.8$ & $<.001$ \\
Ventilation support & 5.5 & $3.8-16.8$ & .003 \\
Preoperative non-SR & 2.1 & $1.3-3.3$ & .002 \\
Surgical procedure & 2.7 & $1.7-4.6$ & .002 \\
Late mortality & & & \\
Age & 1.05 & $1.04-1.07$ & $<.001$ \\
Active Endocarditis & 2.05 & $1.38-3.06$ & $<.001$ \\
Hypertension & 2.39 & $1.56-3.68$ & $<.001$ \\
Severe renal disease & 3.61 & $1.75-7.45$ & .001 \\
Ventilation support & 3.06 & $1.71-5.48$ & $<.001$ \\
Preoperative non-SR & 1.65 & $1.32-2.08$ & $<.001$ \\
Surgical procedure & 1.11 & $1.04-1.18$ & .001 \\
Urgency & 2.88 & $1.75-4.75$ & $<.001$ \\
\hline
\end{tabular}

$H R$, Hazard ratio; $C I$, confidence interval; non-SR, nonsinus rhythm.
$<.001$

.003

.001

.002

002

$<.001$

$<.001$

$<.001$

.001

$<.001$

.001

$<.001$ 


\section{APPENDIX E4. STATISTICAL MODEL BUILDING FOR MIXED-EFFECTS MODELS}

Likelihood ratio test was used for the fixed part and the mixture distribution of $\chi^{2}$ for the random part. We used linear time for aortic gradient $(P=.9302)$, Annulus diameter $(P=.1391)$ and nonlinear for left ventricle end systolic diameter $(P<.0001)$ for the fixed and the random effects. For the ordinal longitudinal outcome linearity was assumed due to convergence problems. Furthermore, we used clinically relevant baseline covariates in the fixed part, namely for left ventricle end systolic, aortic gradient, and annulus diameter we included: sex, left ventricle function, hypertension, etiology of disease, and patient age at operation. For aortic valve regurgitation, we included sex, left ventricular function, hypertension, diagnosis, and etiology. Finally, no serial correlation term in the residual errors was assumed because it could result in estimation problems.

\section{Specification of Mixed-Effects Models and Joint Models of Longitudinal and Survival Data}

Let $T_{i}^{*}$ denote the true failure time for the i-th individual ( $\mathrm{i}=1, \ldots, \mathrm{n})$, and $\mathrm{d} C_{i}$ the censoring time, then $T_{i}=\min \left(T_{i}^{*}, C_{i}\right)$ represents the observed failure time for the $\mathrm{i}$-th patient. Moreover, $\delta_{i}=0,1 \mathrm{i}$ is the event indicator where 0 indicates censoring.

For the longitudinal part, we let $y_{i}$ consist of longitudinal responses that may be obtained at different time points $t_{i j}$ and have length $n_{i}$. To describe the subject-specific evolutions over time of the longitudinal outcome we use a linear mixed-effects model. Specifically, it takes the form,

$$
y_{i}(t)=f_{i}(t)+\varepsilon_{i}=x_{i}^{T}(t) \beta+z_{i}^{T}(t) b_{i}+\varepsilon_{i},
$$

where $x_{i}(t)$ denotes the design vector for the fixed effects regression coefficients $\beta$ and $z_{i}(t)$ the design vector for the random effects $b_{i}$.

Finally, we assume that a normal distribution for the random effects describes the evolution of the longitudinal outcomes, ie,

$$
b_{i} \sim N\left(0, \sigma_{b}\right),
$$

Where $\sigma_{b}$ is the variance of the random intercept.

For the survival process we have:

$$
h_{i}\left(t, \theta_{s}\right)=h_{0}(t) \mathrm{e}^{\left\{\gamma^{T} \omega_{i}+\alpha f_{i}(t)\right\}},
$$

where $\theta_{\mathrm{s}}$ is the parameter vector for the survival outcomes, $\omega_{\mathrm{i}}$ is a vector of baseline covariates with a corresponding vector of regression coefficients $\gamma$, and $\alpha$ denotes the strength of association between the longitudinal and survival outcomes. Moreover, a Weibull baseline hazard $\mathrm{h}_{0}(\mathrm{t})=\psi \mathrm{t}^{\psi-1}$ was assumed.

Syntax for one joint model. Variable notation in the model:

Gender(male of female $)=$ Sex

Left ventricle function $=\mathrm{LV}$ function

Patient age at operation $=$ ptageatok

Hypertension $=$ Hypertension

Etiology of disease $=$ Etiology

Echotime $=$ ' time of echocardiogram

Annulusdiameter $=$ diameter of annulus

MaxOfFUP $=$ maximum follow-up time

Active.endocarditis $=$ Active endocarditis

VentilationSupport $=$ Ventilation support

Type.of.operation $=$ subcoronary or full root

Urgency.code.for.operation = Urgent $(<24$ hours $)$, within the same hospitalization or elective

FUPreop $=$ follow-up time for reoperation

Reop $=$ reoperation

library (JM)

fm1 $<-$ lme(Annulusdiameter $\sim$ echotime + Sex + LVfunction + Hypertension + Etiology + ptageatok.x, data $=$ data, na.action $=$ na.exclude, ,andom $=\sim$ echotime $\mid$ IDnr $)$ coxFit.avD. <- $\operatorname{coxph}$ (Surv(MaxOfFUP, LastOfDeath.) $\sim$ Active.endocarditis + Hypertension + VentilationSupport + Type.of.operation + Urgency.code.for.operation, data $=$ data.id, $\mathrm{x}=$ TRUE)

coxFit.avR. <- $\operatorname{coxph}(\operatorname{Surv}($ FUPreop, Reop) $\sim$ Hypertension + Etiology + Sex + LVfunction, data $=$ data.id, $\mathrm{x}=$ TRUE)

jointFit.avD $<$ - jointModel(fm1, coxFit.avD., timeVar $=$ "echotime", method = "piecewise-PH-aGH", verbose $=$ TRUE, iter.EM = 80)

summary(jointFit.avD)

jointFit.avR <- jointModel(fm1, coxFit.avR., timeVar $=$ "echotime", method = "piecewise-PH-aGH", verbose $=$ TRUE, iter.EM $=80$ )

summary(jointFit.avR)

Additional software package:

Joint modeling of longitudinal and survival data; Package: JM (version: 0.8-3) 
APPENDIX E5. Allograft statistics

All patients $(n=353)$

Subcoronary technique $(n=92)$

Root replacement $(n=261)$

Type of allograft

Aortic

$$
\begin{gathered}
98 \%(\mathrm{n}=348) \\
2 \%(\mathrm{n}=5)
\end{gathered}
$$
$22.7(2.1 ; 14-30)$
$84 \%$
$16 \%$

$40(13 ; 8-62)$

Mean (SD; range)

Preservation method

Cryopreserved

Fresh
$95 \%(\mathrm{n}=88)$

$5 \%(n=4)$
$23.3(2.4 ; 19-30)$
$70 \%$
$30 \%$

$36(13 ; 12-60)$

$94 \%$

$6 \%$
$>99 \%(\mathrm{n}=260)$

$<1 \%(\mathrm{n}=1)$

$22.4(1.9 ; 14-28)^{*}$

$89 \%$

$11 \%$

$42(13 ; 8-62)^{*}$

$>99 \%$

$<1 \%$

$S D$, Standard deviation. *Statistical significant difference between the 2 groups (unpaired $t$ test or Mann-Whitney $U$ test)

Aortic valve regurgitation over time

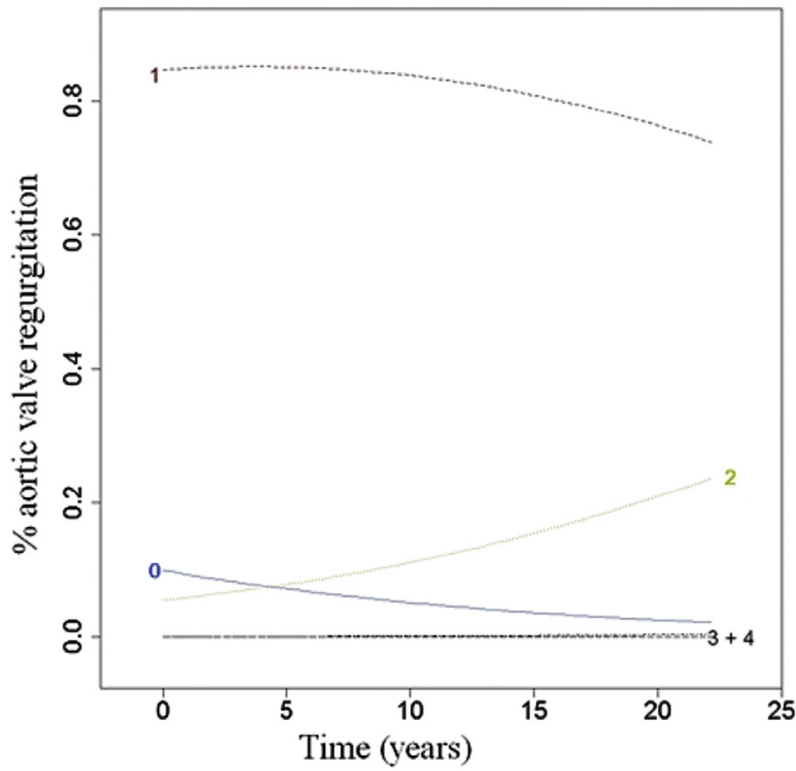

APPENDIX E6. Progression of aortic valve regurgitation (AR) grade during years. A mixed effect model was used to predict variables associated with progression of $\mathrm{AR}$ grade. Blue line indicates grade $0 \mathrm{AR}$, red line grade $1 \mathrm{AR}$, green line grade $2 \mathrm{AR}$, and black lines grade 3 and $4 \mathrm{AR}$. 